Hamburg

\title{
IST SANDHI EIN FIKTIVES ODER EIN REALES LINGUISTISCHES PROBLEM?*
}

Es ist nicht so einleuchtend wie es auf den ersten Blick scheinen mag, daß es neben der etablierten Segmentphonetik auch eine Satzphonetik geben soll. Gegenstand der Satzphonetik wäre in erster Linie die Beschreibung der Übergänge zwischen Segmenten, die vorher von der Segmentphonetik bestimmt und beschrieben worden wären. Die Satzphonetik wäre also auf jeden Fall von der Segmentphonetik abgeleitet.

Dieser Standpunkt setzt voraus, daß die Eigenschaften der Segmente bekannt sind, denn andernfalls können keine Übergangserscheinungen zwischen Segmenten erfaßt und beschreiben werden. Aber hier ist der Kern einer noch nicht hinreichend geklärten Frage. Wo finden wir diese Segmente, deren Eingeschaften die Segmentphonetik in ihrer Beschreibung festlegt? Diese Segmente finden wir weder im akustischen Signal noch im artikulatorischen Bewegungskontinuum. Auf keiner der beiden Ebenen gibt es Invarianten, die wir als Segmente identifizieren können, d.h. Eigenschaften, die für einen bestimmten Laut bzw. Lauttyp konstant bleiben. Das ist aber noch kein Beweis dafür, daß Segmente als Invarianten auf höherem, der Forschung noch nicht zugänglichem, Niveau vorkommen. Solche Argumentation löst aber keineswegs das Problem, sondern versetzt es nur auf ein anderes Niveau.

Aus dieser Problemlage ergibt sich, daß entweder die Segmente nicht existieren, da sie sich weder auf der akustisch-physikalischen noch auf der artikulatorischen Ebene nachweisen lassen, oder daß sie auf den beiden genannten Ebenen zwar existieren, aber der Forschung nicht zugänglich sind. Im ersten Falle ist Sandhi ein nicht-existentes und daher ein fiktives Problem. Da es keine Segmente gibt, gibt es nur Dauerbewegungen, die die einzige phonetische Wirklichkeit darstellen. Aus dieser Perspektive wäre es nur die Satzphonetik, die reale Existenz hätte.

Dieser Standpunkt läßt sich aber nicht durchsetzen. Die Variations-möglichkeiten sind unendlich und sie erlauben keinerlei Systematisierung, die erkennbare Beziehung zur Sprache als menschlichem Kommunikationsmittel hätte. Das besondere Kennzeichen der Sprache ist, daß sie eine hierarchische Struktur aufweist, in der bedeutungsdifferenzierende Elemente die bedeutungstragenden Elemente bilden. In beiden Fällen handelt es sich um diskrete Einheiten. Die Laute werden als

* Dies ist das Manuskript meines Vortrages auf dem "Tenth International Congress of Phonetic Sciences“, Utrecht, 1.-6. August 1983 
Realisierung von Phonemen festgelegt, die aufgrund der Kommutationsprobe durch Minimalpaare und aufgrund anderer seit langem in der Linguistik erprobter Kriterien ermittelt werden. Gerade diese Beziehungen akustischer und artikulatorischer Ereignisse zur Semantik stellen das Charakteristikum der Phonetik dar, die sich dadurch von der reinen Physiologie und von der reinen Akustik unterscheidet. Da diskrete Segmente im Signal nicht vorhanden sind, kann das nur bedeuten, daß das Segment vor der Signalanalyse bzw. vor der Signalerzeugung existiert und vom Forscher mit festen Attributen bzw. festen Eigenschaften in das Signal ${ }^{1}$ hineinprojeziert wird. Wenn nun von solchen Segmenten mit wohl definierten Attributen ausgegangen wird, erhält man einerseits die Laute der traditionellen Segmentphonetik und andererseits die Grundlage der Satzphonetik, die darin besteht, zu untersuchen, wie die den einzelnen Segmenten zugwiesenen Attribute sich beim Lautübergang verhalten. Die Satzphonetik setzt deshalb das Vorhandensein der Segmentphonetik voraus, welche den Ausgangspunkt der satzphonetischen Untersuchung bildet. Nur dann ist Satzphonetik sinnvoll, wenn sie von solider segmentphonetischer Beschreibung ausgeht. Sandhi definiert als phonetische Modifikation von Lauten, zwischen denen eine Wortgrenze verläuft, ist nur dann eine reale phonetische Erscheinung, wenn vorher Segmente mit klar definierten Eigenschaften etabliert worden sind. Andernfalls ist Sandhi ein fiktives und inexistentes Problem.

Wir vertreten den segmentphonetischen Standpunkt und betrachten Sandhi unter dieser Voraussetzung als reales Problem. Die Frage muß aber gestellt werden, wo die Segmente reale Existenz haben. Auf diese Frage hat die Phonetik noch keine Antwort. Es läßt sich nur vermuten, daß das Segment im Gehirn geschaffen wird, aber wie und unter welchen Voraussetzungen das geschieht, ist völlig unbekannt.

- Diesen Tatbestand hatte die klassische Phonetik schon längst erkannt. Er erklärt einerseits die Reserven der linguistisch orientierten Phonetiker gegen die experimentelle Phonetik und andererseits die Tatsache, daß zu den Beschreibungen phonetischer Norm einer Sprache und zu der phonetischen Transkription in jeder Sprache Realisierungshinweise zugefügt werden müssen. Denn Laute mit den klar definierten Attributen einer Segmentphonetik kommen in der tatsächlich gesprochenen Sprache kaum vor. Die segmentphonetischen Beschreibungen beziehen sich häufig nur auf isoliert ausgesprochene Laute, die im Sprechen kaum je unter solchen idealen Bedingungen vorkommen können. Statt der idealen Laute kommen im Sprechen Allophone in fast unendlicher Anzahl vor. Die Form der Allophone hängt von einer Menge nicht vollständig erfaßter Faktoren wie z.B. Lautumgebung, Betonung, Intonation, Sprechgeschwindigkeit und Stilebene ab. Genau genommen tritt jedes Allophon nur einmal auf. Aber dieses nur ein einziges Mal vorkommende Allophon steht im Kontrast zu anderen Allophonen in der syntagmatischen Kette. Dal Allophon ist in einer solchen Sprachkonzeption ein diskretes Element auf syntagmatischer Ebene, das Resultat eines ersten Abstraktionsprozesses, eine diskrete Einheit ersten Grades.

1 Auch der Hörer identifiziert Segmente mit festen Attributen, obwohl ihm die Attribute der einzelnen Segmente nicht bewußt bekannt sind. 
Demgegenüber ist das Phonem als diskrete Einheit der paradigmatischen Ebene von der Lautumgebung unabhängig, bildet aber distinktive Oppositionen maximal effektiv. Da das Allophon per definitionem kontextabhängig ist, kann es die distinktive Funktion nicht im vollen Umfang ausüben. Deshalb ist es notwendig zwischen dem Sprechen und der Sprache eine Zwischenstufe anzunehmen, auf der die distinktiven Laute vorkommen, die die klar definierten Attribute der Laute der traditionellen Segmentphonetik besitzen. Diese Zwischenstufe gehört weder dem Sprechen noch der Sprache voll an. Auf der Ebene des Sprechens üben diese Einheiten (Laute) nun kontrastive Funktion in der Gestalt nur einmal vorkommender Allophone aus, aber auf der Ebene der Sprache üben sie simultan distinktive Funktion aus. Schematisch kann man des folgendermaßen darstellen:

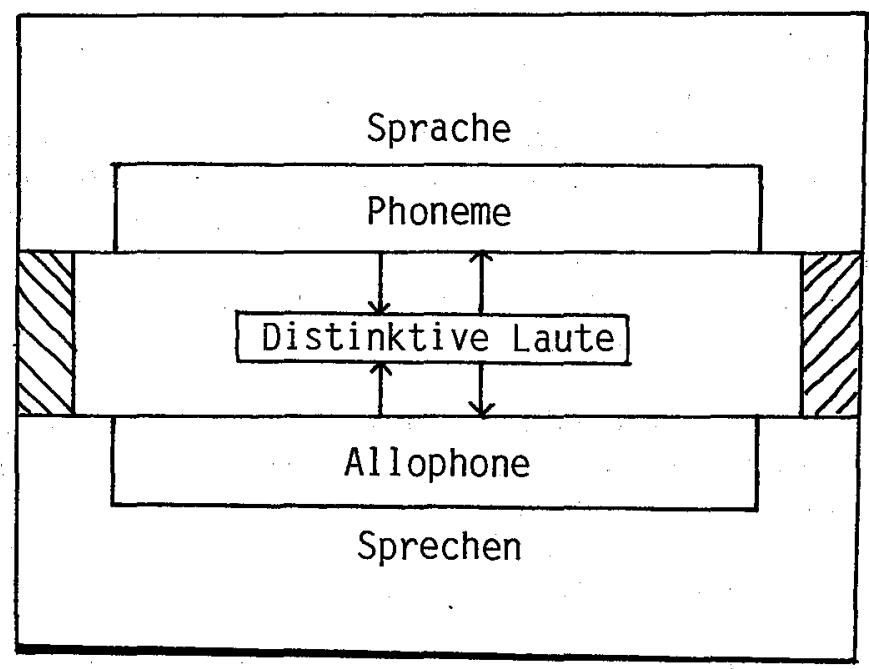

Es folgt aus solchem dreischichtigen Model der Sprache, daß im Sprechen Distinktivität nicht durch rein materielle Erscheinungen, die mit dem effektiv realisierten Laut identisch wären, ausgeübt werden kann. Distinktivität wird durch Einheiten realisiert, die eine Unmenge materieller Formen aufweisen, und die im realen Sprechen nur in kontrastiver Funktion auftreten. Diese Einheiten, die auf syntagmatischer Ebene nur verdeckt oder versteckt auftreten, sind die Phoneme.

Aber im Sprechen gibt es nicht nur Kontrastivität, sondern auch Angleichung. Wenn es auf syntagmatischer Ebene nur Kontrastivität gäbe, wäre es unverständlich, daß verschiedene distinktive Einheiten durch Assimilationsprozesse identisch werden können. Das ist nur möglich, weil die auf syntagmatischer Ebene vorkommenden Einheiten auf paradigmatischer Ebene weiterhin unterschieden werden. Die distinktive Eigenschaft des Phonems ist daher eine permanente Eigenschaft, aber daș bedeutet nicht, daß das Nicht-Distinktive irrelevant oder redundant sei. Im Gegenteil, alles, was normalerweise in der sprachlichen materiellen Substanz vorkommt, 
ist für die Kommunikation relevant, mindestens in dem Maße wie es zur Natürlichkeit der Sprache beiträgt (Martinet 1973, pp. 23-24; Jakobson and Waugh 1979, p. 29). Laut dieser Auffassung ist Sandhi nicht nur eine lautliche Erscheinung des Sprechens, sondern hat notwendigerweise wichtige Implikationen für die Sprache. Es ist aber offentsichtlich, wenn zwischen Sprache und Sprechen wenig oder gar nicht unterschieden wird, daß die Sandhi-Erscheinungen die Allophongrenzen überschreiten und den distinktiven Charakter des Phonems beeinflussen. Das scheint der Standpunkt von André Martinet zu sein: “Or, il faut bien se convaincre que la parole ne fait que concrétiser l'organisation de la langue“ (Martinet 1970, p. 25).

Wir meinen, daß das konsequente Vertreten dieses Standpunktes beinahe zur Verneinung der satzphonetischen Analyse führen würde. Das bedeutet nämlich, daß im syntagmatischen Verlauf jeder Laut als solcher erkennbar wird und bleibt, obwohl er gelegentlich durch Nachbarsegmente modifiziert wird. Aber durch solche Modifikation wird er zu keinem neuen Segment. Laute dieser Art, wie z.B. nasalierte Vokale, entstimmte Konsonanten, sonorisierte Konsonanten, labialsierte Laute usw., die gelegentlich durch Modifikation und Einwirken von Nachbarsegmenten entstehen, sind zwar meßbar nachzuweisen, werden aber nicht bewußt vom Sprecher wahrgenommen (Lehiste and Shockey 1974). Die Segmente werden weitherhin dem richtigen Phonem zugeordnet und es gibt keinerlei Grund für eine Linguistik des Sprechens (oder des "parole").

Eine streng positivistische Haltung wäre die Auffassung, daß nur die physikalische Wirklichkeit tatsächlich existiert. In solcher Auffassung gibt es kein Sandhi, weil jeweils nur das konkrete Pänomen an einem bestimmten Ort des syntagmatischen Verlaufs existiert. Die Zahl der Einheiten wird unendlich und Begriffe wie Silbe, Sprachlaut und ähnliche werden gegenstandslos.

Solche streng positivistische Auffassung ist ein Mythos. Es ist unmöglich, etwas mit voll offenem Geist und absoluter Objektivität zu untersuchen, ohne vorher darüber gewisse Vorannahmen gemacht zu haben. Eine solche Vorannahme ist; daß es Segmente gibt, denn ohne solche Vorannahme kann der Positivist sich nicht an die Analyse des Signals begeben. Eine ganze Reihe solcher Vorannahmen fliessen z.B. unbewußt in die Konstruktion der Instrumente ein, mit denen das Signal registriert wird. Sandhi ist nur ein reales linguistisches Problem, wenn von der Auffassung der Sprache als etwas vom Sprechen unterscheidbarem und von Segmenten bzw. distinktiven Lauten ausgegangen wird. Andernfalls ist Sandhi ein fiktives Problem, Sandhi ist nicht eine objektiv vorgegebene Erscheinung, sondern folgt aus dem theoretischen Ansatz, unter dem die Sprache analysiert wird.

\section{SANDHI UND KOARTIKULATION}

Koartikulation ist die natürlichste Erscheinung der gesprochenen Sprache. Kaum eine Äusßerung, sei sie auch nur so klein und kurz, ist ohne Koartikulation 
möglich. Als physiologische Notwendigkeit erklärt die Koartikulation sich daraus, daß die Bewegungsgeschwindigkeit einzelner Sprechorgane und Organteile stark veränderlich und naturgemäß unterschiedlich ist. Es ist daher notwendig, einerseits die Bewegungsgeschwindigkeit der einzelnen Organe auszugleichen und andererseits das Zusammenwirken unterschiedlicher Organe zu koordinieren. Liberman (1981, p. 111) meint, daß diese Funktionsweise der Sprechorgane daraus in erster Linie zu erklären ist, daß es sich um Organe in sekundärer Funktion handelt. Wenn die erzeugten Lautsegmente im Signal diskret und nicht koartikuliert wären, würde das bedeuten, daß die Sprechorgane sehr schnelle und zerhackte Bewegungen auszuführen hätten. Aber Organe, die in solcher Art funktionieren würden, würden für die vitale Funktion unbrauchbar sein. Koartikulation ist daher physiologisch notwendig; aber ihre perzeptorischen Effekte werden im Minimum gehalten, da sie eine Erscheinung des Sprechens ist und nicht die Organisation der Sprache beeinflußt. Diese Feststellung steht in keinerlei Widerspruch zu der bekannten Tatsache, daß bestimmte Transitionen die Perzeption von bestimmten Konsonanten begünstigen. Nicht die Transition wird wahrgenommen, sondern das diskrete Segment, dessen phonetische Merkmale die Transition beinhandelt (Cooper, Delattre, Liberman, Borst, Gerstman 1952; Delattre, Liberman, Cooper 1955; Kuehn, Moll 1974).

Da Koartikulation in der Natur der menschlichen Sprache liegt, ist es auch zu erwarten, daß die potentiellen Merkmale eines phonetischen Segments im Signal verteilt sind und mit Merkmalen anderer Segmente gemischt werden. Es ist daher zu erwarten, wie das auch der Fall ist, daß Merkmale mehrerer Segmente simultan realisiert werden. Die Integration solcher Merkmale auf mehreren Ebenen ist vermutlich die Strategie, die zur Perzeption diskreter Einheiten in Form von Sprachlauten beiträgt. Wie diese Strategie im einzelnen verläuft, ist aber bisher weitgehend unbekannt.

Man kann Koartikulation nicht als potentielle oder effektive Lautveränderung betrachten, denn dazu müste Effekt über das Sprechen hinausgehen und in das Sprachsystem hineingreifen. Bislang kann aber keine Theorie den Zeitpunkt bestimmen, an dem die Koartikulationsvorgänge die Allomorphie erreichen und daher das Sprachsystem erfassen. Erst wenn das geschehen ist, wird es möglich von einer Lautveränderung zu sprechen.

Auch wenn Koartikulation an Sandhi-Erscheinungen beteiligt ist, ist Sandhi mehr als Koartikulation. Wir gehen hier nicht auf die phonetische Form von Sandhi-Erscheinungen ein, die sich von totaler Assimilation bis zur Entstehung neuer Laute und Lautkombinationen mit zahlreichen Zwischenstufen sowohl auf segmentaler als auch auf suprasegmentaler Ebene streckt (Pétursson 1980; Pilch 1981). Uns: geht es in erster Linie darum festzustellen, daß Sandhi das Sprachsystem erreicht (Allen, 1972, p. 15), während Koartikulation auf der Ebene des Sprechens als mechanischer Faktor bleibt.

Sandhi entsteht rein mechanisch gesehen aus den Zwängen, denen die zeitliche 
Organisation der Sprache unterliegt. Diese Zwange hat Ernst A. Meyer wohl als erster nachgewiesen: "Die vokaldauer im zweisilbigen wort ist gegenüber der im einsilbigen wort durchweg beträchtlich gekürzt“ (Meyer 1902-1904, p. 82). Die Forschung har Meyers Entdeckung später eindrucksvoll bestätigt (siehe die Zusammenfassung in Lehiste 1970). Lehistes Untersuchungen scheinen zu beweisen, daß diese organisierte Zeiteinheit der Sprache ("foot" in ihrer Terminologie) nicht von der morphologischen und lexikalischen Struktur bestimmt wird (Lehiste 1975, p. 117). Wenn das der Fall ist, ist offensichtlich, daß Sandhi als Anpassung an diese Zeitkonstanten der sprache entsteht, die sich je nach Sprechgeschwindigkeitändern, da die zur semantischen Einheit gehörigen Morpheme innerhalb eines bestimmten Zeitrahmens realisiert werden müssen. Um das zu erreichen, muß eventuell die lautliche Form geändert werden. Sandhi ist hier ein Instrument, das es ermöglich, die Morpheme lautlich zu ändern, so daß sie sich dem Zeitrahmen anpassen. Besonders deutlich sind solche Tendenzen in einer Suffixsprache wie z.B. im Japanischen, das eindrucksvolle Beispiele dieser Art aufweisen kann. Solche in Sandhi entstandene Allomorphe können eventuell lexikalisiert werden, so daß ihre ursprüngliche Form kaum erkennbar ist.

Sandhi hat morphologische Implikationen. Sandhi ist im gleichen Maße morphologisch und phonetisch, aber Koartikulation ist nur phonetisch (auch wenn sie über Wortgrenzen hinausgeht), ohne die Morphologie und damit das Sprachsystem zu berühren. Deshalb ist Sandhi die Quelle neuer Lautmöglichkeiten und Lautverbindungen der Sprache, weil neue Laute und Lautkombinationen durch Sandhi sprachliche bzw. distinktive Funktion übernehmen, durch die sie dem Sprachsystem angehören und durch die sie in das Sprachsystem integriert werden. Sandhi kann deshalb die Möglichkeiten eines. Sprachsystems aufzeigen, die die betreffende Sprache auf eine ihr eigene Art als Substanz in Lautveränderungen verwenden kann. Sandhi bleibt daher ein reales linguistisches Problem.

Phonetisches Institut der Universität Hamburg

\section{BIBLIOGRAPHIE}

Allen, W. Sidney. 1972. Sandhi. Mouton: The Hague.

Cooper, F. S.; Delattre, P. C.; Liberman, A. M.; Borst, J. M. and Gerstman; L. J. 1952. Some experiments on the perception of synthetic speech sounds. Journal of the Acoustical Society of America 24, 597-606.

Delattre, P. C.; Liberman, A. M. and Cooper, F. S. 1955. Acoustic loci and transitional cues for consonants. Journal of the Acoustical Society of America 27, $769-773$.

Jakobson, Roman and Waugh, Linda R. 1979. The sound shape of language. Bloomington and London: Indiana University Press. 
Kuehn, D. P. and Moll, K. L. 1974. Perceptual effects of forward coarticulation. Experimental Phonetics, ed. by Norman J. Lass, 237-247. New York: MSS Information Corporation.

Lehiste, Ilse. 1970. Suprasegmentals. Cambridge (Mass.): MIT Press.

Lehiste, Ilse. 1975. The role of temporal factors in the establishment of linguistic units and boundaries. Phonologica 1972, ed. by. Wofgang U. Dressler and F. V. Mareš, 115-122. München: Wilhelm Fink Verlag.

Lehiste, Ilse and Shockey, Linda. 1974. On the perception of coarticulation effects in English VCV syllables. Experimental Phonetics, ed. by. Norman J. Lass, 230-236. New York: MSS Information Corporation.

Liberman, Alvin M. 1981. On finding that speech is special. Status Report on Speech Research 67/68 (Haskins Laboratories), 107-143.

Martinet, André. 1970. Eléments de linguistique générale. Paris: Armand Colin.

Martinet, André. 1973. La pertinence. Journal de Psyshologie Normale et Pathologique, 19-30 (Fascicule 1-2).

Meyer, Ernst A. 1902-1904. Englische Lautdauer. Eine experimentalphonetische Untersuchung. Uppsala: Skifter utgivna af Humanistiska Vetenskapssamfundet i Upssala, 8:3.

Péturson, Magnús. 1980. Quelques formes intonatives indiquant pauses en islandais. Arkiv för Nordisk Filologi 95, 189-195.

Pilch, Herbert. 1881. Weltsprache Englisch in Forschung und Lehre. Festschrift für

Kurt Wächtler, hrsg. von Peter Kunsmann und Ortwin Kuhn, 221-232, Berlin: Erich Schmitt Verlag.

Povzetek

JE SANDHI IZMIŠLJENO ALI RESNIČNO JEZIKOSLOVNO VPRAŠANJE?

Sandhi ni objektivno dan pojav, temveč ga pojmujemo, kakor narekuje vsakokratni teoretični pristop $\mathbf{k}$ razčlembi jezika. Ce priznamo obstoj razlikovalnih glasovnih segmentov — in to po piscu moramo -, je sandhi resnično jezikoslovno vprašanje. Po svoji naravi se razlikuje od koartikualcije v tem, da sodi slednja $v$ 'parole', sandhi pa posega $v$ jezikovni sestav in se včasih širi iz glasoslovja in fonologije $v$ oblikoslovje. Sandhi pogojuje specifična časovna organizacija jezika. 